\title{
Numerical and experimental investigation on the effects of bumps over the leading edge of the micro wind turbine blades suitable for low wind regimes
}

\author{
Viswanathan $\mathrm{P}^{1}$,Prabu $\mathrm{T}^{2}$, Jayachitra $\mathrm{R}^{3}$, Sivasubramaniam $\mathrm{S}^{4}$, Archana $\mathrm{J}^{5}$ \\ \{vp.mech@psgtech.ac.in $\left.{ }^{1}\right\}$ \\ tpr.mech@psgtech.ac.in ${ }^{2}$, rjr.mech@psgtech.ac.in ${ }^{3}$, s.sivasubramaniam@gmail.com ${ }^{4}$, \\ archanaj@bitsathy.ac.in ${ }^{5}$ \\ ${ }^{1}$ Faculty, ${ }^{2}$ Scholar, ${ }^{3}$ Student \\ Department of Mechanical Engineering, PSG College of Technology
}

\begin{abstract}
Wind Energy has become one of the important energy sources for many countries as it safe guards the environment from pollutionMicro wind turbines are wind turbines that produce less than 1000 watts of power and are mostly utilised for residential purposes in urban areas. The National Institute of Wind Energy(NIWE)had stated that in India, wind speeds rangesbetween 0 to $5 \mathrm{~m} / \mathrm{sin}$ the urban areas. The wind-turbine blades are the critical component that converts the energy available in the wind. Micro-turbine blades should be developed to suit the wind conditions present in a given location. To boost power conversion, particular features like as vortex generators, bumps, and slats have been introduced to the blades in recent years.A delayed stall and a higher lift coefficient were the results of the adjustments. The "S1223" blade, which had a narrow airfoil and a high lift coefficient, was investigated in this article. It was originally designed for low-reynolds-number carrier aircraft. The blades were modified with bumps on the leading edge, similar to those of a humpback whale, to increase aerodynamic performance characteristics. The numerical calculations were performed on a 2-D blade spline and a 3-D rotor, with the results compared between the blades with standard and curved airfoil sections...Rapid prototyping is utilised to build numerically evaluated 3-D rotor models, which are then tested in a wind tunnel for wind turbine system operational performance. The redesigned blade section had an $11 \%$ increase in coefficient oflift, while the prototype rotor study revealed a $20 \%$ increase in torque over the blade section with a normal airfoil. In wind tunnel experiments, the modified rotor with the lower cutin speed produced positive results. As a result, it's evident that in a low-wind, urban environment, micro-turbines with modified blades will theoretically produce 75 percent more energy than rotors with regular blades., Micro-turbines with modified blades should produce $75 \%$ more energy than rotors with standard blades.
\end{abstract}

Keywords: bionic blade, aerodynamics, bio-mimicry, bumps, S1223, micro wind turbine, airfoil. 


\section{Introduction}

In today's atmosphere, wind energy has proven to be a lifesaver for many countries. Pollution free energy has become priority for harvesting energy from all available sources for all. In May 2017, the capacity of wind energy systems installed reached $511 \mathrm{GW}$. According to the report of International Energy Agency, the total power generation was 24,255 TWh in 2015, the wind energy accounting for $838 \mathrm{TWh}, 3.5$ percent of total electricity generation[1]. The ability to generate electricity is dependent on the availability of strong winds.. The windy areasare classified as Class I to Class IV, depending on the available wind speed.There are a limited number of high-wind Class I and moderate-wind Class II sites.

The vast majority of India's land is covered by Class III and Class IV wind, which has a wind speed of less than $5 \mathrm{~m} / \mathrm{s}$ and a wind power potential of less than 200 Watts per square metre at 50 metres above sea levelaccording to the NIWE. Urban regions,are densely populated the amount of power available in these cities are limited, which can be used directly if properly harnessed, lowering the power generated from harmful conventional sources. Micro turbines or small turbines are the best systems suitable for these locations. These systems' energy can be used for either household or captive uses. Micro-turbines must be particularly efficient in this wind environment. At low Reynolds numbers, micro-turbine blades should have outstanding aerodynamic performance features like a high coefficient lift, low coefficient drag, well adhered boundary layer flow, delayed stall, and so on.To boost performance, vortex generators, bionic bumps, slats, and other characteristics are incorporated into the blades (Lin and John C, [3], Quackenbush T R et al., [4], Von Stillfried F, [5].

In this paper, the performance ofaerodynamic characteristics of a thin airfoil S-1223, blade extruded normally versus blade incorporated with bionic bumps similar to humpback whale fins on the leading edge, are compared, and the feasibility of using the blade for turbines of urban environment is investigated. Due to several constraints, wind energy is more difficult to harness in metropolitan settings. In India, where space for wind structures is restricted or non-existent in urban areas, the overall system's weight should be extremely light so that the structures do not interfere with buildings or dwellings. The whole system weight should be very light if put on the roof top so that the structures do not damage the buildings or dwellings. The reactionary forces of the rotating system should be kept to a minimum to allow a house with titled structures to stand; the turbine's diameter should not exceed 2 metres. The generated acoustic noise should not bother adjacent inhabitants, and the wind speed in this location is less than $5 \mathrm{~m} / \mathrm{s}$. For these environmentmicro-turbines are the best devices since they can generate small amounts of electricity (less than 1000 Watts) suitable for lights and other low-power applications.

Microturbines designed for these conditions should have a lower cut-in speed, produce high torque at low wind speeds, and produce the specified power at the wind speed available. The turbine's blades, and hence its aerofoils, must have a high lift coefficient, delayed stall, and low drag coefficient to meet the design requirements. For a large range of wind speeds, the operating characteristics are flat.

\section{BIOMIMICRY}

Biomimicry is a new design method that draws inspiration from nature. Natural biological processes and systems were used to produce the design solutions. Traditional systems have 
seen significant advancements thanks to the adaption of nature's technique [6]. The goal of developing blades for wind turbines was to see whether there were any improvements that could be made to traditional blade designs by incorporating natural elements. The humpback whale flippers characters were studied and imitated on wind turbine blades to improve performance. The banking turns are quite sharp and quick. Biomimicry is a new design method that draws inspiration from nature.Natural biological processes and systems were used to produce the design solutions. Traditional methods have seen significant breakthroughs as a result of the adoption of nature's technique [6]. The goal of creating wind turbine blades was to see whether there were any that could be made better. Improvements could be accomplished by making appropriate alterations to standard blade designs. The humpback whale prefers tubercles that have a high lift-to-drag ratio and flippers with a high aspect ratio.Bumps on the leading edge of the humpback whale's flippers, also known as tubercles, are a sinusoidal pattern found on the whale's flippers. The addition of the flipper function to the leading edge of the blades eliminates stall and improves lift.

\subsection{Bionic Blades}

H T C Pedro and M H Kobayashi compared the scalloped flippers of a humpback whale leading edge blade to a standard conventional edged blade in anunmanned air vehicles and studied the performance [7]. The low Reynolds number effect the sort of separation in the flippers, and the aerodynamic performance is doubled. Z Carija et al. studied the aerodynamic effects of sinusoidal bumps on the NACA 0012 blade section and found that the bumped blade have a high lift-to-drag ratio advantage over a angle of attack (AoA) from $0^{\circ}$ to $20^{\circ}[8]$. A K Malipeddi, N Karthikeyan et al, studied the effects of leading edge tubercles on the separation of laminar bubble on NACA 2412 aifoil.[9,10]

The laminar bubble that existed in the baseline airfoil at $6^{\circ}$ angle of attack did not exist in the modified airfoil with leading edge tubercles, and the flow separation point was moved from $10 \%$ of chord for the baseline airfoil to $60 \%$ downstream for the modified airfoil [11]. ACorsini et al., quantitatively analyzed the profiles of NACA4415 and NACA0015, both normal and with hump wave leading edge and predicted $30 \%$ rise in lift with a stabilizing impact on the suction side.Also the occurrence of separation was solely at the trailing edge in span wise cross sections, which was correlated to leading edge troughs[12]. P Viswanathan et al. analytically analysed and compared the NACA 0012 blade with and without bumpsand observed that the blade with bumps provided 24 percent more lift than the conventional blade for increased AoA up to 25 degrees[13].

\section{Airfoil Geometry}

Tiny or micro turbines with shorter blades provide small amounts of power in the $\mathrm{kW}$ range in urban wind systems. The high-rise structures, along with other buildingsin the city, blocks the path of wind, limiting wind speed dramatically. As a result, wind forces on small/micro wind turbine blades are smaller than on large wind turbine blades. Thin-section airfoils can now be investigated as a result. Thin airfoil blade sections may require less initial torque than thick airfoil blade sections due to their lower mass. Wind turbine blades with S1223 airfoil and bionic modification are researched to see if they are suitable for micro turbines.

M Selig devised the S1223 airfoil to achieve maximum lift at a Reynolds number of 200k by combining the advantages of both concave pressure recovery and aft loading. The S1223 
airfoil has a maximum lift coefficient of 2.11 at a 16-degree angle of attack. [14]. The ordinates distribution of the S1223 airfoil is shown in Figure 1.

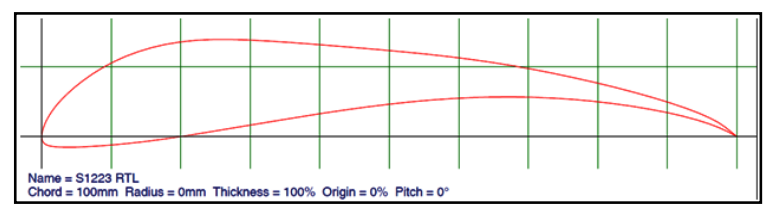

Figure 1 S 1223 Airfoil

The bionic enhancement is achieved by adding bumps to the blade's leading edge. The bumps are made by alternately protruding and caving by $10 \%$ in the chord from the leading edge of a standard blade. A sinusoidal wave pattern's pitch is equal to $30 \%$ of the chord's length. The amplitude and wave length sizes were determined using an experiment by D Custodio et al., on leading edge protuberances on limited span wings. The study found that the protuberance produced a $10 \%$ higher lift coefficient than the baseline blade span with the NACA 634-021 airfoil[15]. In the redesigned blade geometry, the wavelength of the sine wave shaped leading edge is equal at every portion of the blade. The surface area of the standard conventional blade and the boincally modified, bumped blade are kept the same for better comparisons. The baseline and modified blade section 2D span models are shown in Figure 2. Table 1 shows the geometry and attributes of the blades that were evaluated for numerical simulation..

Table 1 Comparison between the base line and the humpback modified blade sections of S 1223

\begin{tabular}{|l|l|l|l|}
\hline $\begin{array}{l}\text { S1.N } \\
\text { o. }\end{array}$ & Description & $\begin{array}{l}\text { Base line } \\
\text { blade } \\
\text { model }\end{array}$ & $\begin{array}{l}\text { Modified } \\
\text { blade model }\end{array}$ \\
\hline 1 & Chord (mm) & 100 & $\begin{array}{l}\text { Max-110 } \\
\text { Min- 90 }\end{array}$ \\
\hline 2 & Span (mm) & 100 & 100 \\
\hline 3 & $\begin{array}{l}\text { Surface area } \\
\left(\mathrm{m}^{2}\right)\end{array}$ & 0.021 & 0.021 \\
\hline 4 & No of elements & 565268 & 535248 \\
\hline 5 & No of nodes & 597860 & 556860 \\
\hline
\end{tabular}

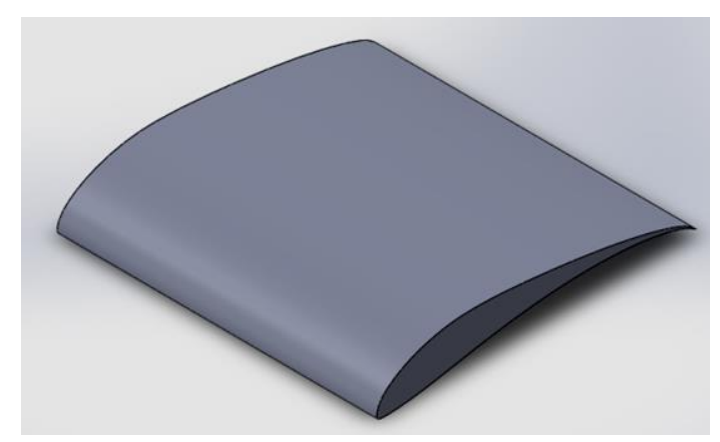


(a) Baseline blade section

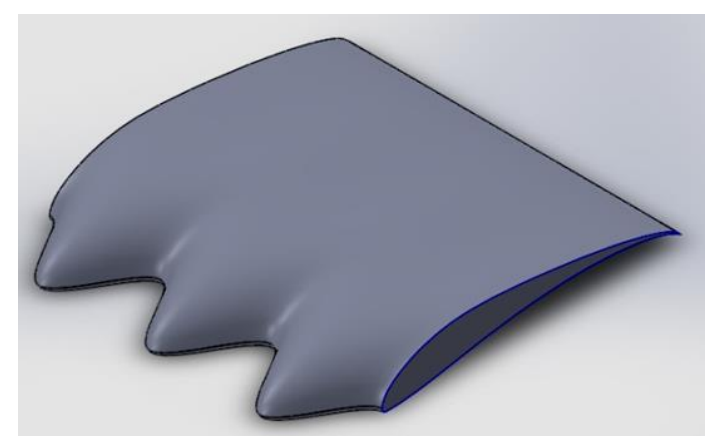

(b) Modified blade section

Figure 2 2D Span wise blade sections

\section{NUMERICAL EVALUATION OF BLADE SPANS}

Ansys code is used to calculate incompressible, laminar / turbulent, 2-dimensional / 3dimensional, steady / unsteady flows and is compatible with a variety of processors, the 'Ansys - Fluent' software is used for numerical analysis. Some of the researchers who have successfully used Fluent to solve flow problems over airfoils include C PMellen et al., [16], H Beri et al., [17], DEleni et al., [18], and K zGharali et al., [19].

The Reynolds Averaged Navier Stokes two equation model 'k - $\omega$ turbulence model' (Equation 1-5) is preferred for solving the flow[20]. In the equation, where $\mathrm{k}$ is the turbulent kinetic energy and $\omega$ is the specific rate at which the turbulent kinetic energy is dissipated into internal thermal energy, the two partial difference equations for two variables, $\mathrm{k}$ and, predict turbulence in the equation, where $\mathrm{k}$ is the turbulent kinetic energy and is the specific rate at which the turbulent kinetic energy is dissipated into internal thermal energy. Modifications for low-Reynolds number effects, compressibility, and shear flow spreading are included in the kmodel. The model is applied to both wall constrained and free shear flows, and is used to anticipate free shear flow spreading rates that are very close to data for far wakes, mixing layers, plane, round, and radial jets. [18] Eleni D C

$$
\begin{aligned}
& \frac{\partial(\rho k)}{\delta t}+\frac{\delta\left(\rho u_{j} k\right)}{\delta x_{j}}=P-\beta^{*} \rho \omega k \\
& +\frac{\delta}{\delta x_{j}}\left[\left(\mu+\sigma_{k} \frac{\rho k}{\omega}\right) \frac{\delta k}{\delta x_{j}}\right](1) \\
& \frac{\partial(\rho \omega)}{\partial t}+\frac{\partial\left(\rho u_{j} \omega\right)}{\partial x_{j}}=\frac{\gamma \omega}{k} P-\beta \rho \omega^{2} \\
& \quad+\frac{\delta}{\delta x_{j}}\left[\left(\mu+\sigma_{k} \frac{\rho k}{\omega}\right) \frac{\delta \omega}{\delta x_{j}}\right]+\frac{\rho \sigma_{d}}{\omega} \frac{\partial k}{\partial x_{j}} \frac{\partial \omega}{\partial x_{j}}(2) \\
& \quad \text { Where } \\
& P=\tau_{i j} \frac{\partial u_{i}}{\partial x_{j}} \\
& \tau_{i j}=\mu_{i}\left(2 S_{i j}-\frac{2}{3} \frac{\partial u k}{\partial x_{k}} \delta_{i j}\right)-\frac{2}{3} \rho k \delta_{i j} \\
& S_{i j=} \frac{2}{3}\left(\frac{\partial u_{i}}{\partial x_{j}}+\frac{\partial u_{j}}{\partial x_{i}}\right)
\end{aligned}
$$


Second order upwind momentum solutions based on least square cells are employed. If the solution does not converge within 1000 iterations, the iteration count is increased by 1000 steps. The research is done at angles of attack ranging from -5 to 30 degrees, with a 5 degree increment closer to the peak values and a 2 degree increment further away. The study is carried out with a $5 \mathrm{~m} / \mathrm{s}$ input velocity and a Reynolds number of 1105 (Abott and Von Doenhoff 1959) [21].

\subsection{Results and Discussion}

The performance of the blade sections can be compared by plotting non-dimensionalized parameters such as coefficient of lift, coefficient of drag, and pitching moment against various angles of attack and comparing the geometry of the airfoil, the pressure distribution over the chord using equation 6-7. The non-dimensional parameters are simple to comprehend and contrast. M H Sadraey [22] designed the aircraft.

$$
\begin{aligned}
& C_{l}=\frac{L}{\frac{1}{2} \rho C V_{\infty}^{2}} \\
& C_{d}=\frac{D}{\frac{1}{2} \rho C V_{\infty}^{2}} \\
& C_{m}=\frac{m}{\frac{1}{2} \rho C V_{\infty}^{2}}
\end{aligned}
$$

From theplot, lift coefficient $\left(\mathrm{C}_{1}\right)$ versus angle of attack $(\alpha)$, various importantfeatures which describes the airfoil performances such as: 1) the stall angle $\left.\left(\alpha_{s}\right) ; 2\right)$ maximum lift coefficient $\left(\mathrm{C}_{\text {lmax }}\right)$; 3) zero lift angle of attack $\left(\alpha_{\mathrm{o}}\right)$; 4)ideal lift coefficient $\left(\mathrm{C}_{\mathrm{li}}\right)$; 5) angle of attack corresponding ideal lift coefficient $\left(\alpha_{\mathrm{Cli}}\right)$; 6$)$ lift coefficient at zero angle of attack $\left(\mathrm{C}_{\mathrm{lo}}\right)$; 7) lift curve slope $\left(C_{l \alpha}\right)$ can be observed.From theplot,drag coefficient $\left(C_{d}\right)$ to lift coefficient $\left(\mathrm{C}_{1}\right)$, the minimum drag coefficient $\left(\mathrm{C}_{\mathrm{dmin}}\right)$ andthe corresponding minimum lift coefficient $\left(\mathrm{C}_{1}\right.$ $\min )$ can be observed. The optimum angle of attack $(\alpha)$, which is an important feature of the airfoil isalso observed from the plot $C_{1} / C_{d}$ versus angle of attack $(\alpha)$.

\subsection{Coefficient of Lift and Drag}

Figures 3 and 4 illustrate coefficients of lift and drag computed numerically for baseline and modified blade spans at various angles of attack. The coefficient of lift produced for both baseline and changed blade spans follows a similar trend, according to the coefficient of lift plot. Both blade components have a positive lift coefficient at zero degrees AoA. The coefficient of lift increases linearly up to 10 degrees AoA before stalling and for subsequent AoA increases, resulting in lower lift generation.

At 10 degrees AoA, the standard baseline blade generates lift of 1.98 , while the modified blade produces a lift coefficient of 2.2, which is 11.11 percentage more than the normal baseline blade.For the conventional baseline blade, stall occurs immediately after the maximum lift producing angle of attack, whereas for the bionic augmented blade, it can last up to 15 degrees longer. The blade will be able to operate in a wide variety of wind speeds thanks to the stall delay. The drag coefficient increases gradually as the angle of attack increases from 10 degrees, as shown in the drag plot, the drag coefficient increases gradually as the angle of attack increases from 10 degrees for the modified blade, whereas the standard baseline blade has a softer slope up to 20 degrees and increases rapidly. Both the blade sections have the same drag coefficient at the highest lift producing angle of attack, hence the overall power production from the modified blade will be the higher because the lift coefficient is higher for the same drag. 


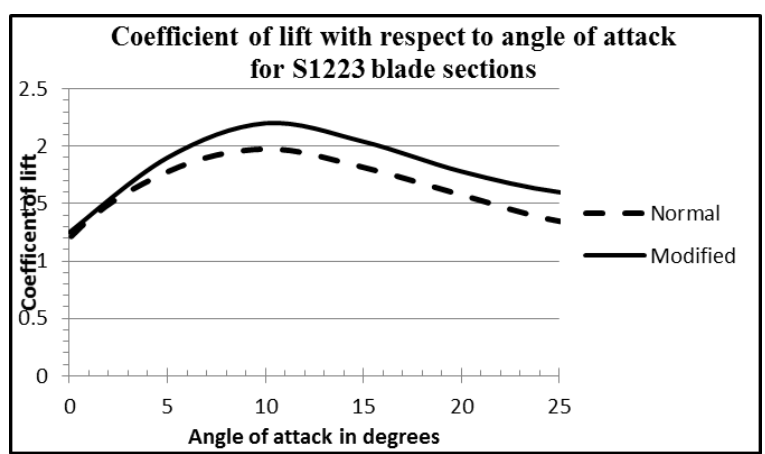

Figure 3 Comparison of Lift coefficient against angle of attack

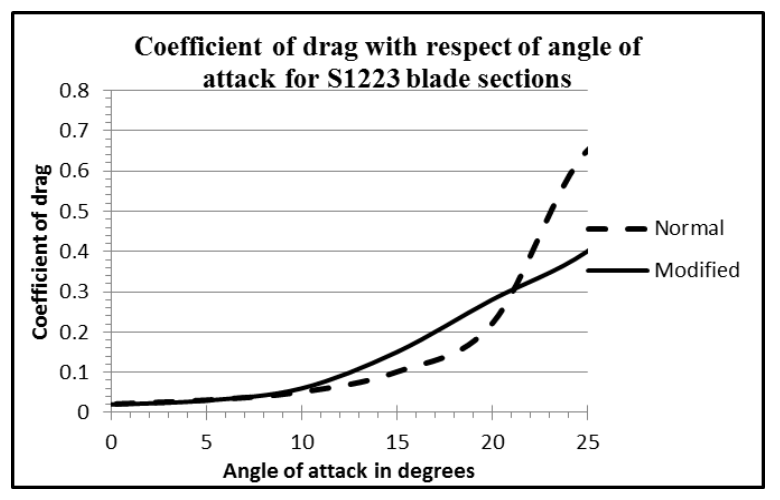

Figure 4Comparison of drag coefficient against angle of attack

\section{NUMERICAL EVALUATION OF 3-D ROTOR BLADES}

The suitability of the airfoil and bionic mimics as a 3-D rotor may be studied further based on the numerical evaluation of two-dimensional blade span. The torque ' $\mathrm{Q}$ ' in equation 10 can be used to calculate the rotors' power output, and the performance of 3-D rotors can be analysed and compared with another non-dimensional metric, the Coefficient of Torque 'CQ' as given in equation 9 .

Power, $P=2 \pi n Q$

$$
C_{Q}=\frac{Q}{\rho n^{2} B^{5}}(9)
$$

A three-bladed rotor is designed to deliver 1,000 Watts of rated power from a wind velocity of $5 \mathrm{~m} / \mathrm{s}$, and the model is scaled down to 1: 10 ratio for computational and experimental research.The rotor diameter of the designed wind turbine is $2300 \mathrm{~mm}$, and that of the model is $230 \mathrm{~mm}$. The bumps on the leading edge of the modified rotor blades are shown in comparison to the standard baseline leading edge rotor blades in figure 5. 


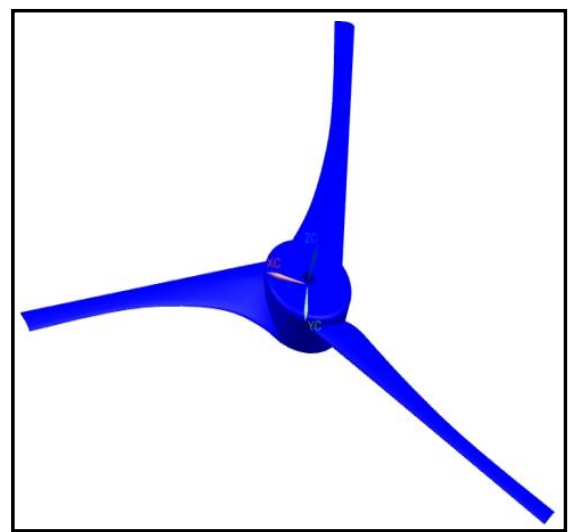

(a) Rotor with normal leading edge

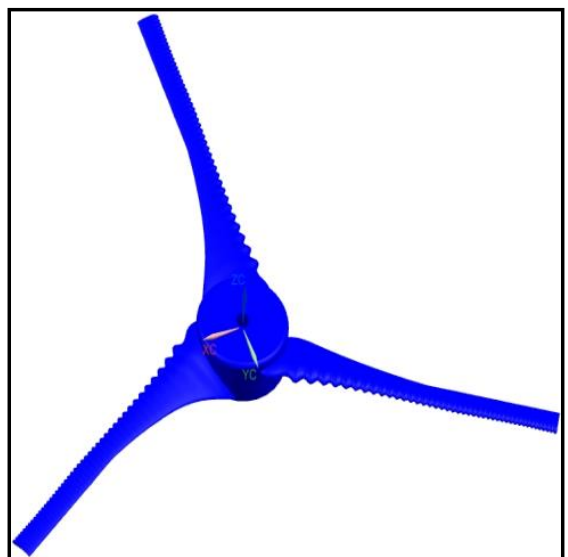

(b) Rotor with bumped leading edge

Figure 5 Rotor with (a) normal and (b) bumped leading edge

The numerical analysis is carried by constructing a local disc over the rotor, The fluid domain surrounding the local disc shaped like a cubical domain to resemble the wind tunnel test section. The size of the cubical domain is 10 times the diameter of the rotor on both sides. An unstructured coarse mesh was used to mesh the local disc and domain. The solution is initialised using a turbulent kinetic energy and dissipation model with a second order upwind approach. The time step is computed using the Courant no, which is initially set to 100 .

The torque coefficients estimated for both normal and modified rotors are shown in Figure 6. The modified rotor torque coefficient ranges from 4 to 3.5 , with an average of 3.7, whereas for the normal rotor torque coefficients vary from 3.5 to 3 , with an average of 3.1. The modified rotor produces $20 \%$ greater average torque than the typical baseline rotor. The rotor will generate more power if the torque coefficient is higher. 


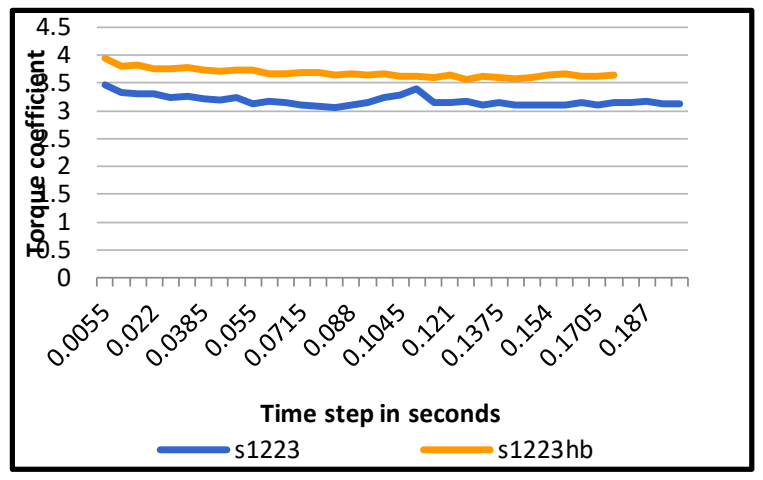

Figure 6Toque coefficients of normal and modified rotors

\section{EXPERIMENTAL EVALUATION OF THE ROTORS}

The bionic modified blade span and the rotor have been demonstrated to result in positive numerical increments, however this needs to be tested in real-world circumstances. The true characteristics of the airfoil profiles, blades, and the turbine rotor as a whole will be revealed by the on-field tests. Another approach is to carry out the tests in a controlled setting, which is where wind tunnels come in helpful. Wind tunnels have been utilised to undertake experimental research with airfoils and wings by M Selig [14], J L Tangler [23], W A Timmer [24], and others.

To validate the rotors, an open wind tunnel with lower turbulence levels is chosen over a closed wind tunnel.The open wind tunnel is an induced draught type, with an axial fan on the diverging end drawing the air from the bell-mouth section. A $5 \mathrm{~kW}$ motor drives the axial fan and the speed of the fan is controlled by a Variac, by which the speed is controlled from from 0 to $2000 \mathrm{rpm}$. The length of the test portion is $310310450 \mathrm{~mm}$. A pitot-static tube inserted at the entering section is used to monitor the velocity of the air going through the test section. In the tunnel, the air velocity can be varied from 0 to 45 metres per second.

Figure 7 depicts the 3 -D rotors that were made in a rapid prototyping machine utilising an ABS fusion depositing method. 


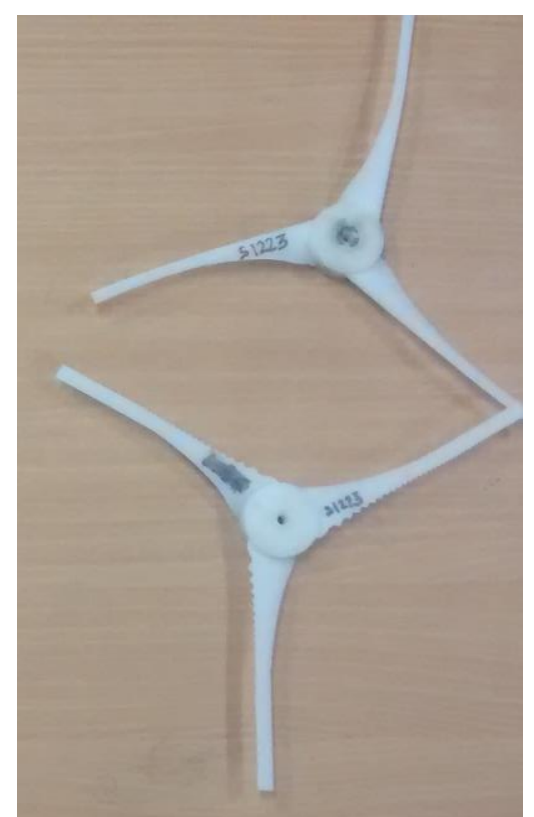

Figure7Fabricated models of normal and bumped modified rotors

\subsection{Experimental setup}

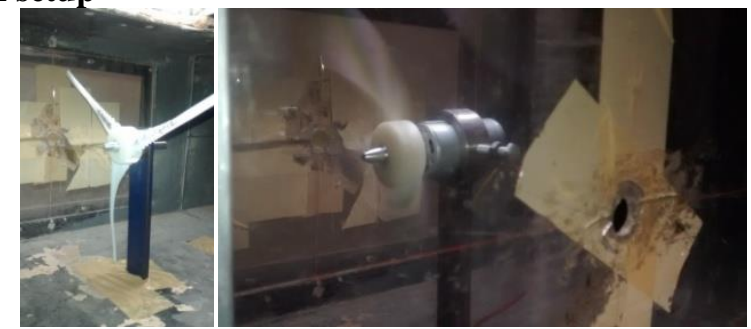

Figure 8 Rotor at wind tunnel testing

Figure 8 shows the rotors, which are supported by a spindle and bearing system from the bottom of the test section.. The prototype rotors are scaled-down models that will not produce any detectable power. They are evaluated for no-load speed at various air velocities. The rotor speed is measured with a non-contact IR tachometer. The air speed is increased in $1 \mathrm{~m} / \mathrm{s}$ increments from 0 to $15 \mathrm{~m} / \mathrm{s}$, then decreased in the same manner from maximum to minimum speed. The experiment is repeated several times to ensure repeatability.

\subsection{Result and discussions}

The experiment yielded results for cut-in speed and rotor speed under various wind speeds. The rotor's performance is measured by comparing the rpm of normal and modified rotors.Figure 9 depicts the observed rotor speeds for various air velocities. 


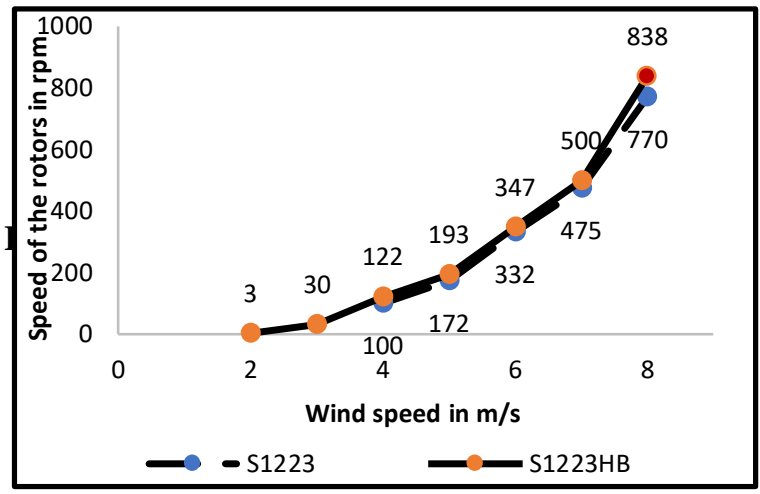

The normal rotor started to rotate at around $4 \mathrm{~m} / \mathrm{s}$ of air velocity, which is known as the rotor's cut-in speed.The speed increased from $100 \mathrm{rpm}$ to $3,080 \mathrm{rpm}$ at $15 \mathrm{~m} / \mathrm{s}$ of air velocity. Above $10 \mathrm{~m} / \mathrm{s}$ of velocity, high flap wise and edge vibrations were observed. The bionic-modified rotor started rotating at $2.2 \mathrm{~m} / \mathrm{s}$, maintained a constant speed of $30 \mathrm{rpm}$ at 3 $\mathrm{m} / \mathrm{s}$, and reached a top speed of $4,100 \mathrm{rpm}$ at $15 \mathrm{~m} / \mathrm{s}$. The bionic rotor produced higher revolutions in comparison to the normal rotor for all air velocities, which is plotted and shown in the figure 9.

The bionic modified rotor's cut-in speed was decreased to $2.2 \mathrm{~m} / \mathrm{s}$ from $4 \mathrm{~m} / \mathrm{s}$, implying that when bionic bumps are introduced to the leading edges of the S1223 rotor, the rotor will attain the requisite design speed at lower wind speeds than standard bladed rotors. This supports the positive results of the blade section and rotor, analyzed through numerical simulations. It is evident that the bionic property of the humpback whale, as demonstrated in the aforementioned experiment, will improve the performance of wind turbine rotors in the field by generating more electricity.

\section{ANNUAL POWER GENERATION}

Equation 11 may be used to compute the average energy produced by wind turbine rotors using the cut-in, rated, and cut-out speeds, and equation 12 can be used to calculate the annual power output in $\mathrm{kWh}[26]$.

$$
P_{e, a v e}=P_{e R}\left\{\frac{\exp \left[-\left({ }^{U_{C} / C}\right)^{k}\right]-\exp \left[-\left({ }^{U_{R} / C}\right)^{k}\right]}{\left(\left({ }^{U_{R} / C}\right)^{k}-\left({ }_{C} / C\right)^{k}\right)}-\exp \left[-\left({ }^{U_{F} / C}\right)^{k}\right]\right\} W
$$




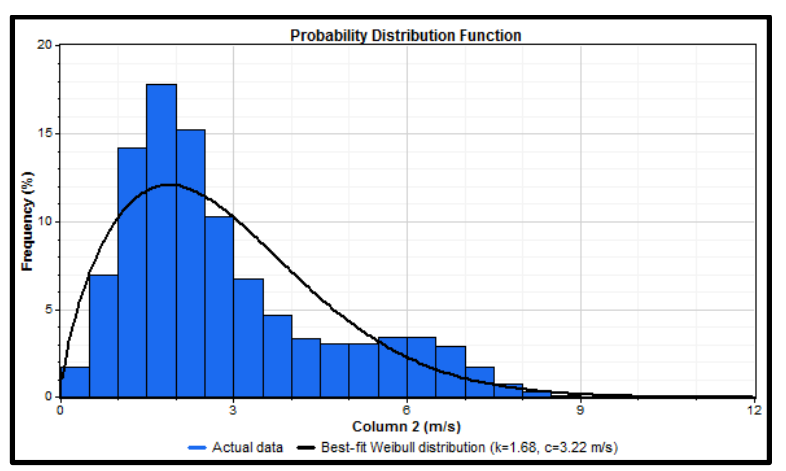

FIG.10. Probability distribution of $10 \mathrm{~min}$ averaged wind speed of Peelamedu, Coimbatore [PV]

$\mathrm{Pe}_{\mathrm{R}}$ is the rated power of the wind turbine, $\mathrm{Uc}$ is the cutting speed of the turbine, $\mathrm{U}_{\mathrm{R}}$ is the speed at which the rated power is created, $\mathrm{U}_{\mathrm{F}}$ is the cut-out or furling speed of the turbine, and $\mathrm{C}$ and $\mathrm{K}$ are the Weibull scale and shape parameters.

Power generation is predicted based on the rotor blades design and experimental characteristics for a sample site for which wind data are available. The wind is continuously monitored and recorded for a 10-minute average using a wind mast in the Peelamedu area of India's Coimbatore district, which is located at $11.0248 \mathrm{~N}$ latitude and $77.0028 \mathrm{E}$ longitude.From the wind data collected, the shape factor ' $K$ ' and scale factor ' $C$ ' for the selected site are computed as 1.68 and $3.22 \mathrm{~m} / \mathrm{s}$, respectively..

The annual power generation of rotors with normal and modified airfoil blades is estimated using experimental data. The cut-in speed $U_{C}$ for the rotors are $4 \mathrm{~m} / \mathrm{s}$ for normal rotors and $2.2 \mathrm{~m} / \mathrm{s}$ for modified rotor. The rated wind speed UR is wind speed at which the turbine will attain the rated revolutions and will generate the rated power. The wind generator is designed to produce $1 \mathrm{~kW}$ of power at a design speed of 300rpm, from the experiment the required rotor speed is achieved at $5.5 \mathrm{~m} / \mathrm{s}$ (figure 9). Equations 11 and 12 are used to calculate the power average $\mathrm{P}_{\text {eave }}$ and yearly power production, and the results are given in Table 2.

Table 2 Annual energy generation by the rotors of S 1223

\begin{tabular}{|l|l|l|}
\hline Rotor & Normal & $\begin{array}{l}\text { HB } \\
\text { modified }\end{array}$ \\
\hline Operating time in hours & 1952 & 3853 \\
\hline $\begin{array}{l}\text { wind speed of the rotors to } \\
\text { produce rated power in m/s }\end{array}$ & 5.5 & 5.5 \\
\hline $\begin{array}{l}\text { Operating hours with rated } \\
\text { output in hours }\end{array}$ & 1388 & 1654 \\
\hline Average Power in $\mathrm{kWm}{ }^{2}$ & 148.5 & 261.3 \\
\hline $\begin{array}{l}\text { Annual energy developed by } \\
\text { the rotors in kWh/m }{ }^{2}\end{array}$ & 1301 & 2288 \\
\hline $\begin{array}{l}\text { Percentage increase in the } \\
\text { energy production by the } \\
\text { modified turbines }\end{array}$ & - & $75 \%$ \\
\hline
\end{tabular}

From table 2 it can be inferred that the operating time of bionic modified rotor increased by 97 percent, which in turn increased the annual power generation by $75 \%$. The study 
found that incorporating bionic feature on the blades of wind turbines improves their performance, allowing them to produce more energy at lower wind speeds.

\section{CONCLUSION}

Numerical and experimental investigations were performed by incorporating bionic humpback whale bumps on the S1223 airfoil. The 2-D numerical simulation of the bionic modified blade showed an 11 percent increase in lift coefficient and a 20 percent increase in torque coefficient over the normal blade and rotor. Experiments in a simulated environment also revealed that modifying the leading edge lowers the cut-in speed while increasing the rpm with respect to air velocities. The bionic modified rotor if installed in a wind turbine will generate 75 percent more energy annually than normal rotors,

Overall, the study revealed that incorporating the bionic feature of humpback whale bumps on the leading edge of the rotor will enhance the performance of the wind turbine that will be used in low wind regimes. Fabrication of blades with and without bumps, field testing, and analysing the actual performance of rotors are all part of the future scope of work.

\section{References}

[1] Statistics, I. "Key world energy statistics 2017." International Energy Agency (2017).

[2] Fuglsang, P., and H. Aagaard Madsen. "Optimization method for wind turbine rotors." Journal of Wind Engineering and Industrial Aerodynamics 80.1-2 (1999): 191-206.

[3] Lin, John C. "Review of research on low-profile vortex generators to control boundary-layer separation." Progress in Aerospace Sciences 38.4-5 (2002): 389-420.

[4] Quackenbush, Todd, Robert McKillip, and Glen Whitehouse. "Development and testing of deployable vortex generators using SMA actuation." 28th AIAA Applied Aerodynamics Conference. 2010.

[5] Von Stillfried, Florian. Computational studies of passive vortex generators for flow control. Diss. KTH, 2009.

[6] Fish, Frank E., Paul W. Weber, Mark M. Murray, and Laurens E. Howle. "The tubercles on humpback whales' flippers: application of bio-inspired technology." (2011): 203-213.

[7] Carreira Pedro, Hugo, and Marcelo Kobayashi. "Numerical Study of stall delay on humpback whale flippers." 46th AIAA Aerospace Sciences Meeting and Exhibit. 2008.

[8] Čarija, Zoran, et al. "Numerical analysis of aerodynamic characteristics of a bumped leadingedge turbine blade." Engineering Review 34.2 (2014): 93-101.

[9] Malipeddi, Anil K., NiloufarMahmoudnejad, and Klaus A. Hoffmann. "Numerical analysis of effects of leading-edge protuberances on aircraft wing performance." Journal of Aircraft 49.5 (2012): 1336-1344.

[10] Gawad, Ahmed Farouk Abdel. "Numerical Simulation Of The Effect Of Leading-Edge Tubercles On The Flow Characteristics Around An Airfoil." International Mechnaical Engineering Congresss \& Exposition. 2012.

[11] Karthikeyan, N., S. Sudhakar, and P. Suriyanarayanan. "Experimental studies on the effect of leading edge tubercles on laminar separation bubble." (2014): 1-16.

[12] Corsini, Alessandro, Giovanni Delibra, and Anthony G. Sheard. "On the role of leading-edge bumps in the control of stall onset in axial fan blades." Journal of Fluids Engineering 135.8 (2013): 081104 . 
[13] Viswanathan, P., Prabu, T., Sivasubramaniam, S., \&Rudramoorthy, R. (2016). AERODYNAMIC PERFORMANCE EVALUATION OF HUMPBACK WHALE INSPIRED WIND TURBINE BLADE USING CFD. Advances and Applications in Fluid Mechanics, 19(4), 837.

[14] Selig, Michael S., and James J. Guglielmo. "High-lift low Reynolds number airfoil design." Journal of aircraft 34.1 (1997): 72-79.

[15] Custodio, Derrick, C. W. Henoch, and Hamid Johari. "Aerodynamic characteristics of finite span wings with leading-edge protuberances." AIAA Journal 53.7 (2015): 1878-1893.

[16] Mellen, C. P., Fr-ograve, J., hlich, \&Rodi, W. (2003). Lessons from LESFOIL project on largeeddy simulation of flow around an airfoil. AIAA journal, 41(4), 573-581.

[17] Beri, H., \& Yao, Y. (2011). Effect of camber airfoil on self starting of vertical axis wind turbine. J. Environ. Sci. Technol, 4(3), 302-312.

[18] Eleni, Douvi C., Tsavalos I. Athanasios, and Margaris P. Dionissios. "Evaluation of the turbulence models for the simulation of the flow over a National Advisory Committee for Aeronautics (NACA) 0012 airfoil." Journal of Mechanical Engineering Research 4.3 (2012): 100-111.

[19] Gharali, K., \& Johnson, D. A. (2013). Dynamic stall simulation of a pitching airfoil under unsteady freestream velocity. Journal of Fluids and Structures, 42, 228-244.

[20] Wilcox, David C. "Formulation of the kw turbulence model revisited." AIAA journal 46.11 (2008): 2823-2838.

[21] I H Abbott and A E von Doenhoff (2015), "Theory of Wing Sections: Including a Summary of Airfoil Data Dover Publications", Dover Publications, Inc., New York.

[22] Sadraey, Mohammad H. Aircraft design: A systems engineering approach. John Wiley \& Sons, 2012.

[23] Tangler, J. L., \& Somers, D. M. (1995), NREL airfoil families for HAWTs, National Renewable Energy Laboratory, pp. 117-123.

[24] Timmer, W. A., \& Van Rooij, R. P. J. O. M. (2003). Summary of the Delft University wind turbine dedicated airfoils. Journal of Solar Energy Engineering, 125(4), 488-496.

[25] Johnson, Gary L., and Dr Gary, "Wind Turbine Power, Energy, and Torque", Wind Energy System, Electrical Edition ed., Prentice-Hall Englewood Cliffs (NJ) (2001): 1-4.

[26] S. Suhas , M.J. Silvister Raju , D.S. Vijayan , "Natural fibre reinforcement experimental study in polymer composite", Materials Today: Proceedings, , https://doi.org/10.1016/j.matpr.2020.12.051 